\title{
Behavioral modeling of nonlinear transfer systems with load-dependent $X$-parameters
}

\author{
Seyed Mohammadamin Moosavi, Christian Widemann, and Wolfgang Mathis \\ Leibniz Universität Hannover, Institut für Theoretische Elektrotechnik Leibniz Universität Hannover, Hanover, Germany \\ Correspondence to: Christian Widemann (widemann@tet.uni-hannover.de)
}

Received: 16 January 2017 - Accepted: 27 March 2017 - Published: 21 September 2017

\begin{abstract}
In this contribution, different approaches based on the $X$-parameters ${ }^{\mathrm{TM}}$ to model the behavior of mismatched nonlinear transfer systems are examined. The $X$-parameters based on the $\mathrm{PHD}^{1}$-principle introduced by Verspecht and Root (2006) as an extension of the well-known $S$-parameters describe nonlinear microwave 2-port-networks under large signal conditions. Using load-pull measurement techniques they can be used for arbitrary load situations. Beside this load-pull approach, in the work of Cai et al. (2015), it is stated that it is sufficient to use one optimized $X$-parameter set for each value of the load reflection coefficient without introducing a large error. In another contribution of Cai and $\mathrm{Yu}$ (2015), this approach is extended to cover the whole smith chart with one optimized $X$-parameter set instead. In this work, these different approaches are compared and brought into question.
\end{abstract}

\section{Introduction}

Nowadays, comprehensive behavioral modeling of nonlinear systems is becoming more important. In this respect, small signal $S$-parameters have been extended to a few approaches of large-signal $S$-parameters as a frequency domain behavioral model for nonlinear multi-ports. Amoung these approaches, the so-called $X$-parameters based on the polyharmonic distortion approach of Verspecht and Root (2006) were made commercially available (Root et al., 2013). Since a complete behavioral modeling of a nonlinear system is not possible, $X$-parameters represent approximately the nonlinear system's behavior around its so-called LSOP ${ }^{2}$. For that purpose, the nonlinear system can be simplified by consid-

\footnotetext{
${ }^{1}$ polyharmonic distortion; (Verspecht and Root, 2006).
}

${ }^{2}$ large signal operating point. ering the existence of only one large signal and the other spectral components as additional small signals that are superimposed linearly (Verspecht and Root, 2006). Originally, the $S$-parameters and $X$-parameters are only valid near the matched load condition, e.g. $Z_{\mathrm{L}}=Z_{0}=50 \Omega$, of the multiport measurement or simulation environment. In reality, perfect matching is not realizable and hence behavioral modeling has to account for that load mismatch. For linear systems, the mismatch at each port of a two- or multi-port can easily be determined with the definition of the load reflection coefficient and its relation to $S$-parameters. Nevertheless, a clear definition of load-dependency of nonlinear systems is usually impossible because the reactive part of the load has different behavior at each multiple of the fundamental frequency in nonlinear systems. However, it is shown in literature (e.g., Ghannouchi and Hashmi, 2013) that theoretically, with appropriate bandpass filtering, load dependency can be defined or measured at each harmonic of the fundamental frequency by passive load-pull method. In this work, different approaches to handle load dependency of $X$-parameters are examined and compared. For that purpose, the paper is organized as follows. In Sects. 2 and 3, nonlinear scattering variables and load dependency in both linear and nonlinear systems are recapitulated. In Sect. 4, the load-pull $X$-parameter approach of Root et al. (2013, Sect. 5.2) and simplifying approaches by Cai et al. (2015) and Cai and Yu (2015) are presented. In Sect. 5, the simulation results for each approach based on harmonic balance simulations in Cadence Spectre are discussed.

\section{Nonlinear scattering variables}

Since more than 50 years engineers use $S$-parameters to measure and simulate circuit characteristics. The $S$-parameters 
of linear systems are based on the scattering variables which are power related and thus measurable for radio frequencies in contrast to voltage and current. Mathematically, they are a linear combination of the port voltages and currents (Heaviside transformation) and can be calculated employing complex AC calculations. In many electrical circuits linear conditions do not exist anymore since the spectrum of the port currents and voltages exhibit harmonic distortion and intermodulation. Hence, $S$-parameters are only valid for small signal levels for which the additional spectral components are negligible. Under large-signal excitation with one or more signals at integers of a fundamental frequency $l \omega_{0}$, i.e. there is no intermodulation, the nonlinear two-port's currents and voltages can be formulated in terms of complex Fourier-series (Howell, 2001) assuming their periodic form and neglecting sub-harmonics

$i_{p}(t)=\sum_{k=-\infty}^{\infty} I_{p k} e^{j k \omega_{0}}, u_{p}(t)=\sum_{k=-\infty}^{\infty} U_{p k} e^{j k \omega_{0}}$.

For real signals the complex Fourier-coefficients have to fulfill the condition $I_{p(-k)}=I_{p k}^{*}$ and $U_{p(-k)}=U_{p k}^{*}$, respectively. Since it is a linear operation, the scattering variables in nonlinear multi-ports can be calculated for each harmonic and each port by the Heaviside transformation

$A_{p k}=\frac{U_{p k}+Z_{0, p} I_{p k}}{2 \sqrt{\mathcal{R}\left\{Z_{0, p}\right\}}}, B_{p k}=\frac{U_{p k}-Z_{0, p}^{*} I_{p k}}{2 \sqrt{\mathcal{R}\left\{Z_{0, p}\right\}}}$

where $p$ refers to the port and $k$ to the harmonic of the incident $A_{p k}$ and scattered waves $B_{p k}$, respectively. $Z_{0, p}$ are the characteristic impedance of the port $p$.

\section{Load dependency and mismatch}

As above mentioned mismatch occurs when the load at port $p$ is not equal to the port's characteristic impedance $Z_{\mathrm{L}, p} \neq Z_{0, p}$. In linear systems, the available power is located at the excitation frequency. In fact, the available and obtained power at each port power can be described in a direct relation to scattering variables (Gonzalez, 1997). The load reflection coefficient is defined as ratio of the incident scattering signal $A_{2}$ to the reflected scattering signal $B_{2}$

$\Gamma_{\mathrm{L}}=\frac{A_{2}}{B_{2}}=\frac{Z_{\mathrm{L}}-Z_{0}}{Z_{\mathrm{L}}+Z_{0}}$.

However, in nonlinear systems the clear definition of $\Gamma_{L}$ is more complicated due to the harmonic distortion in the signals spectra as shown in Eq. (2). Nevertheless, in literature, e.g. Ghannouchi and Hashmi (2013), the harmonic reflection coefficients can be found

$A_{2 l}=\Gamma_{2 l} B_{2 l} \forall l \in \mathbb{N}$

which can be formed to a set
$\Gamma_{\mathrm{L}}=\left\{\Gamma_{21}, \Gamma_{22}, \ldots\right\}$.

In the next section, $X$-parameter approaches considering this load reflection coefficients are discussed.

\section{$4 \quad X$-parameters}

In linear multi-ports, $S$-parameters can describe a linear relation between the scattering variables at the ports. However, in nonlinear multi-ports, a multi-dimensional describing function can be defined

$B_{p k}=F_{p k}\left(A_{11}, A_{12}, \ldots, A_{21}, A_{22}, \ldots,\right)$

representing the nonlinear relationship between the incident scattering variables $A_{q l}$ and the reflected scattering variable $B_{p k}$.

By means of a Taylor-series expansion of Eq. (5) around the LSOP, the describing functions convert to the linear (with respect to the small signals $\left.A_{q l}\right) X$-parameter

$$
\begin{aligned}
B_{p k} & \approx X_{p k}^{(\mathrm{F})}\left(\left|A_{11}\right|\right) P^{k}+\sum_{q l} X_{p k ; q l}^{(\mathrm{S})}\left(\left|A_{11}\right|\right) A_{q l} P^{k-l} \\
& +\sum_{q l} X_{p k ; q l}^{(\mathrm{T})}\left(\left|A_{11}\right|\right) A_{q l}^{*} P^{k+l} \forall(q, l) \neq(1,1) .
\end{aligned}
$$

Therein, $A_{q l}$ are the additional small-signals and $P=e^{j \arg \left\{A_{11}\right\}}$. These $X$-parameters depend only on the DC-bias parameters of the circuit, the fundamental frequency $\omega_{0}$ and $\left|A_{11}\right|$ (Verspecht and Root, 2006). However, if the load is not matched as discussed in the previous section the whole transmission power could not be obtained from the load (Gonzalez, 1997). Hence, a part of the power will be reflected. In Root et al. (2013) load-pull approach is introduced to describe the mismatched situation in nonlinear systems.

As a result of the load mismatch, the $X$-parameters depend not only on the input signal $A_{11}$ but also on the reflected large signals due to load mismatch. Therefore, for analyzing these load-dependent $X$-parameters another approach will be needed.

\subsection{Load-pull LSOP-approach}

Root et al. (2013) assume that in addition to $A_{11}$ the reflected signal at the fundamental frequency $\left(A_{21}=\Gamma_{21} B_{21}\right)$ is much larger than the other incident signals $\left(A_{q l} \forall l>1\right)$. Consequently, the describing functions of the system depend not only on the fundamental excitation at the input $A_{11}$ but also on the signal $A_{21}$ caused by reflection due to load mismatch. In other words, the system has two large signals, $A_{11}$ and $A_{21}$. Therefore, the validity of the behavioral model Eq. (6) gained in a matched condition is restricted to a small area on the smith chart. 
Load-dependent $X$-parameters can be determined by active load-pull or passive load-pull measurements or simulations. The meaning of active load-pull can be understood by the corresponding $\mathrm{LSOP}_{\text {active }}=\left\{\mathrm{DCOP}^{3}, \omega_{0},\left|A_{11}\right|\right.$, $\left.A_{21}\right\}$, which is extended by the large signal $A_{21}$. Supposed that this signal can be extracted from load mismatch regarding relation Eq. (4b) due to a mismatched passive load at port 2, the LSOP converts to the passive load-pull $\mathrm{LSOP}_{\text {passive }}=\left\{\mathrm{DCOP}, \omega_{0},\left|A_{11}\right|, \Gamma_{21}\right\}$. Applying the spectral linearization of Eq. (5) in the passive load-pull LSOP leads to the modified $X$-parameter model

$$
\begin{aligned}
B_{p k} & \approx X_{p k}^{(\mathrm{F})}\left(\left|A_{11}\right|, \Gamma_{21}\right) P^{k} \\
& +\sum_{q=1}^{N} \sum_{l=2}^{l_{q, \max }} X_{p k ; q l}^{(\mathrm{S})}\left(\left|A_{11}\right|, \Gamma_{21}\right) A_{q l} P^{k-l} \\
& +X_{p k ; q l}^{(\mathrm{T})}\left(\left|A_{11}\right|, \Gamma_{21}\right) A_{q l}^{*} P^{k+l} \\
& +X_{p k ; 21}^{(\mathrm{S})}\left(\left|A_{11}\right|, \Gamma_{21}\right)\left[A_{21}-\Gamma_{21} X_{21}^{(\mathrm{F})}\left(\left|A_{11}\right|, \Gamma_{21}\right)\right] P^{k-1} \\
& +X_{p k ; 21}^{(\mathrm{T})}\left(\left|A_{11}\right|, \Gamma_{21}\right)\left[A_{21}-\Gamma_{21} X_{21}^{(\mathrm{F})}\left(\left|A_{11}\right|, \Gamma_{21}\right)\right]^{*} P^{k+1}
\end{aligned}
$$

wherein the $X$-parameter depend on $\left|A_{11}\right|$ and complex reflection coefficient $\Gamma_{21}=\left|\Gamma_{21}\right| e^{j \Theta_{21}}$. Therefore, the $X$ parameters $X_{p k}^{(\cdot)}\left(\left|A_{11}\right|,\left|\Gamma_{21}\right|, \Theta_{21}\right)$ in Eq. (7) depend on three parameters what increases the necessary measurements to receive the model enormously.

\subsection{Approaches of Cai}

In the works of Cai et al. (2015) and Cai and Yu (2015), it is noted that the amount of necessary model parameters can be reduced compared to the load-pull $X$-parameters presented in the previous section. The presented approach of Cai et al. (2015) uses one optimized parameter set for each value of the reflection coefficients' magnitude $\left|\Gamma_{21}\right|$. Thus, the $X$-parameters are found for the so-called parametric largesignal operating circle PLSOC $=\left\{\mathrm{DCOP}, \omega_{0},\left|A_{11}\right|,\left|\Gamma_{21}\right|\right\}$, instead of an LSOP.

$$
\begin{aligned}
B_{p k, \mathrm{PLSOC}} & \approx X_{p k}^{(\mathrm{F})}\left(\left|A_{11}\right|,\left|\Gamma_{\mathrm{L}}\right|\right) P^{k} \\
& +\underbrace{\sum_{n=1}^{N} \sum_{l=1}^{L_{n}} X_{p k, n l}^{(\mathrm{S})}\left(\left|A_{11}\right|,\left|\Gamma_{\mathrm{L}}\right|\right) A_{n l} P^{k-l}}_{\{n, l\} \neq\{1,1\}} \\
& +X_{p k, n l}^{(\mathrm{T})}\left(\left|A_{11}\right|,\left|\Gamma_{\mathrm{L}}\right|\right) A_{n l}^{*} P^{k+l} \\
& +X_{p k, n l}^{\left(\mathrm{S}^{2}\right)}\left(\left|A_{11}\right|,\left|\Gamma_{\mathrm{L}}\right|\right) A_{n l}^{2} P^{k-2 l} \\
& +X_{p k, n l}^{\left(\mathrm{T}^{2}\right)}\left(\left|A_{11}\right|,\left|\Gamma_{\mathrm{L}}\right|\right)\left(A_{n l}^{*}\right)^{2} P^{k+2 l} \\
& +X_{p k, n l}^{(\mathrm{ST})}\left(\left|A_{11}\right|,\left|\Gamma_{\mathrm{L}}\right|\right)\left|A_{n l}\right|^{2} P^{k}
\end{aligned}
$$

The possible loss of accuracy by this model order reduction is compensated by increasing the order of the series expan-

\footnotetext{
${ }^{3}$ Direct current operating point; (Cai et al., 2015).

${ }^{3}$ Parametric large-signal operating circle; (Cai et al., 2015).
}

sion underlying the $X$-parameter model. Instead of a linear a quadratic approach is employed and denoted as QPHD ${ }^{4}$. This approach is shown with an adapted notation in Eq. (8) wherein the $X$-parameters $X_{p k}^{(\cdot)}\left(\left|A_{11}\right|,\left|\Gamma_{21}\right|\right)$ depend only on two parameters in addition to the DCOP and the fundamental frequency $\omega_{0}$.

Continuing this idea of model order reduction in Cai and Yu (2015), one optimized parameter set of the QPHD-model approach is extracted for the whole load plane. Thus, every $X$-parameter $X_{p k}^{(\cdot)}\left(\left|A_{11}\right|\right)$ of this approach is a function of the large signal amplitude $\left|A_{11}\right|$ as for the matched case in Eq. (6). For each of the named approaches, it is shown in the following section how the model extraction is performed.

\subsection{Model extraction}

In order to extract the $X$-parameters $X_{p k}^{(\cdot)}$ of the matched Eq. (6) and load-pull Eq. (7) approach, there are two measurement approaches summarized in Root et al. (2013, Sect. 4.4) namely the offset-frequency and -phase technique. The idea of the latter is captured by the approaches of Cai.

In the original offset-phase method, the parameters $X_{p k}^{(\mathrm{F})}$ are found measuring the large signal response only. The small signal $X$-parameters $X_{p k, q l}^{(\mathrm{S})}$ and $X_{p k, q l}^{(\mathrm{T})}$ are found by solving a linear system of equations. If it is over-determined, the impact of measurement outliers can be reduced. The difference between the previously shown approaches is how the datapoints of the system of equations are measured. The offset-phase method (Root et al., 2013) superimposes to each LSOP the small signals $A_{q l}$ separately with different phases $\arg \left\{A_{q l}\right\}$. The PLSOC approach of Cai et al. (2015) sweeps the phase $\Theta_{2 l}, l \geq 1$ of the $l$ th harmonic load-pull tuner for a fixed magnitude $\Gamma_{2 l}$ (cf. Eq. 4b). As a result, there is a randomized change in $A_{2 k} \forall k \geq 1$ due to the reflection Eq. (4b) at the load port along the PLSOC. The full smith chart optimization technique introduced in Cai and $\mathrm{Yu}$ (2015) uses the datapoints of several PLSOCs in order to find one optimized $X$-parameter set for the whole smith chart. This randomized phase variation is not capable to vary the small signals of the input port $A_{1 k}$.

If the large signal parameters $X_{p k}^{(\mathrm{F})}$ are included in the optimization problem, the over-determined system of equations with $M$ data points for all approaches has to be solved e.g. by means of least-squares approximation. The system of the $(\mathrm{Q}) \mathrm{PHD}$-approach is

$$
\left(\begin{array}{cccc}
1 & A_{q l, 1} & A_{q l, 1}^{*} & \ldots \\
\vdots & \vdots & \vdots & \vdots \\
1 & A_{q l, M} & A_{q l, M}^{*} & \ldots
\end{array}\right)\left(\begin{array}{c}
X_{p k}^{(\mathrm{F})} \\
X_{p k, q l}^{(\mathrm{S})} \\
X_{p k, q l}^{(\mathrm{T})} \\
\vdots
\end{array}\right)=\left(\begin{array}{c}
B_{p k, 1} \\
\vdots \\
B_{p k, M}
\end{array}\right)
$$

which can be rewritten as

\footnotetext{
${ }^{4}$ Quadratic polyharmonic distortion; (Cai et al., 2015)
} 


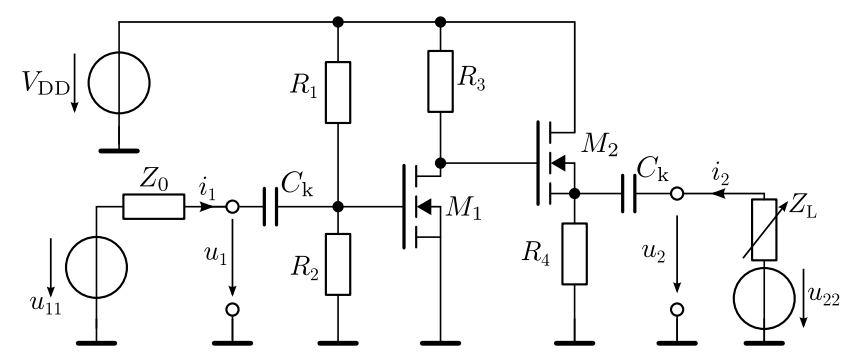

Figure 1. Class-C MOS-amplifier with source follower output stage.

$\mathbf{A} \cdot \mathbf{x}=\mathbf{b}$

with $\mathbf{A} \in \mathbb{C}^{M \times N}, \mathbf{x} \in \mathbb{C}^{N \times 1}$ and $\mathbf{b} \in \mathbb{C}^{M \times 1}$. The small signal excitation matrix $\mathbf{A}$ can be factorized by means of QR decomposition $\mathbf{A}=\mathbf{Q} \cdot \mathbf{R}$ with the orthogonal matrix $\mathbf{Q} \in \mathbb{C}^{M \times N}$ and the upper triangular matrix $\mathbf{R} \in \mathbb{C}^{N \times N}$. Since for orthogonal complex matrices the product with its Hermitian is the unity matrix $\mathbf{I}=\mathbf{Q} \cdot \mathbf{Q}^{\mathrm{H}}$, the system

$\mathbf{R} \cdot \mathbf{x}=\mathbf{Q}^{\mathrm{H}} \mathbf{b}$

results which can be solved by back substitution. Although being slower, the QR decomposition is preferable to the normal equations used by Cai et al. (2015) and Cai and Yu (2015) due to its better numerical properties (Dahmen, 2008).

\section{Simulation results}

In this section, the described methods are shown and discussed using an exemplary Class-C amplifier (cf. Fig. 1). It is designed in Cadence Spectre in an Austria Microsystems $350 \mathrm{~nm}$ process. The amplifier is excitated by the large signal $\left|A_{11}\right|=10.6 \mathrm{~m} \sqrt{W}$ which corresponds to an amplitude of $\hat{u}_{11}=150 \mathrm{mV}$ and an additional small signal source at the second harmonic at the output port with the amplitude of $\hat{u}_{22}=7.5 \mathrm{mV}$ that implies the additional small signal $A_{22}$. The system's periodic steady state is simulated using the harmonic balance method in combination with an loadpull block. The magnitude of the reflection coefficient is set to $\left|\Gamma_{21}\right|=0.65$. The load-pull $X$-parameter are calculated for $\Theta_{21}=k \cdot 45^{\circ}$ for $k=\{0, \ldots, 7\}$ on the basis of a phase sweep $\phi_{22} \in\left[0,355^{\circ}\right]$ with the stepsize of $5^{\circ}$ by means presented in Eq. (9) using MATLAB. In addition to the PHD-based linear load-pull $X$-parameters, for comparison purpose, the QPHD-approach is calculated for each load-pull LSOP gaining $X_{p k, 22}^{\left(\mathrm{S}^{2}\right)}\left(\left|A_{11}\right|, \Gamma_{21}\right)$ etc.

In contrast, the $X$-parameters of the PLSOC-approach Eq. (8) are calculated for $\left|\Gamma_{21}\right|=0.65$ by the randomized phase sweep method (Cai et al., 2015), i.e. varying the loadpull tuner phase $\Theta_{21} \in\left[0,355^{\circ}\right]$ with a stepsize of $5^{\circ}$.

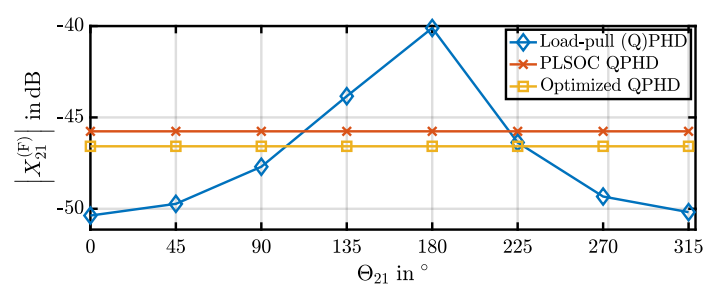

Figure 2. Behavior of magnitude $\left|X_{21}^{(\mathrm{F})}\right|$ swept over $\Theta_{21}$ at $\left|\Gamma_{\mathrm{L}}\right|=0.65$ and $\left|A_{11}\right|=10.6 \mathrm{~m} \sqrt{W}$.

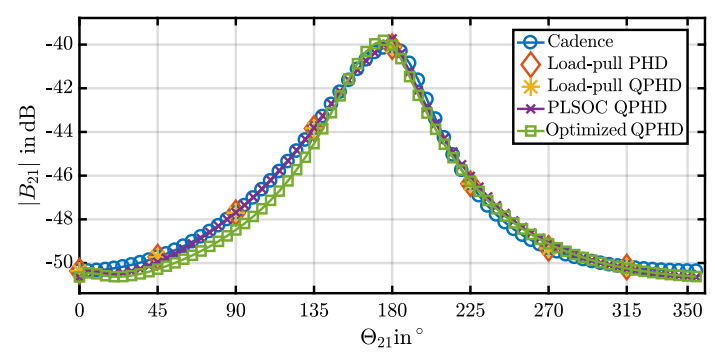

Figure 3. Behavior of $\left|B_{21}\right|$ as a function of $\Theta_{21}$ at $\left|\Gamma_{L}\right|=0.65$.

The optimized QPHD-approach (Cai and Yu, 2015) is calculated by performing the randomized phase sweep for several values of $\left|\Gamma_{21}\right| \in[0.05,0.95]$ with a stepsize of 0.1 .

In Fig. 2, the resulting large signal $X$-parameters $X_{21}^{(\mathrm{F})}$ of each approach are shown over the range of $\Theta_{21}$. The loadpull PHD and QPHD approaches share the same varying non-constant results with a min-max deviation of $10 \mathrm{~dB}$. The PLSOC results in a constant large signal parameter that is approximately in the middle of that variation. The resulting $X_{21}^{(\mathrm{F})}$ of the optimized QPHD-approach lies approximately $1 \mathrm{~dB}$ below that of the PLSOC-approach since it considers measurement values of the whole smith chart. The other not shown $X$-parameter exhibit similar behavior.

The impact of the different $X$-parameters on the scattered variables are shown in Figs. 3 and 4 for the exemplary $B_{21}$. In Fig. 3, the magnitude $\left|B_{21}\right|$ is shown in $\mathrm{dB}$ over the swept variable $\Theta_{21}$. The result shows that the total model calculation of the different approaches in comparison to the cadence simulation. The load-pull $X$-parameters again deliver the same results and map the Cadence simulation. In contrast, the model order reduced approaches namely the PLSOC QPHD and the optimized QPHD exhibit a difference to each other and to the other approaches. In this depiction, the difference seems not that grave. However, shown in the complex plane in Fig. 4, the difference becomes more obvious. As expected, the optimized QPHD which is the most order reduced is not capable of mapping the behavior, whereas the PLSOC seems to be a good compromise.

Nevertheless, the depicted results are shown for the value of the small signal $A_{22}$ that was used for the $X$-parameter extraction which means for which the quadratic error in Eq. (9) was minimized. If the model is used for different values of 


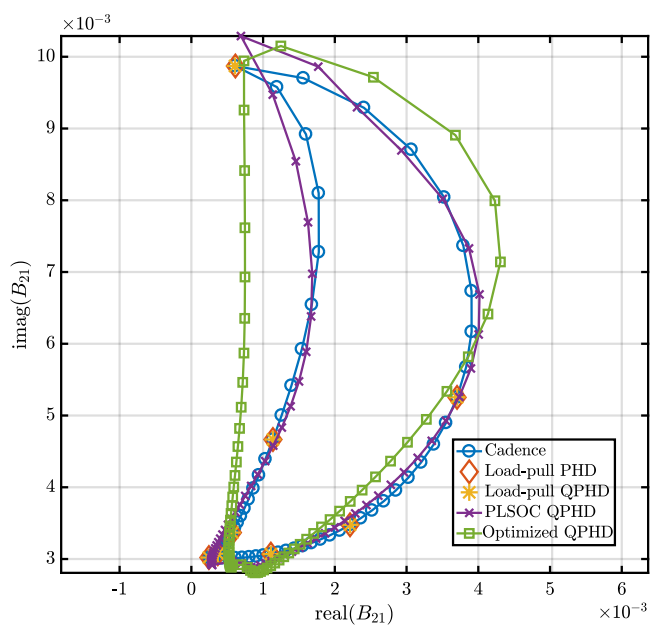

Figure 4. Behavior of $B_{21}$ in the complex plane swept over $\Theta_{21}$ at $\left|\Gamma_{\mathrm{L}}\right|=0.65$.

the small signal, the behavior can differ severely. For the exemplary load situation $\Gamma_{21}=0.65 e^{j 135^{\circ}}$ a sweep over the small signal source up to $\hat{u}_{22}=\frac{\hat{u}_{11}}{2}$ is shown in Fig. 5. The extraction value is marked with a vertical black line. As seen from the previous figures, for that value the models deliver a close result to the reference simulation in Cadence. However, for larger values of the small signal excitation the model order reduced approaches differ severely. Taking a closer look to the behavior of the load-pull PHD- and QPHD-approach, it can be observed that although being of higher order and hence against expectation the QPHD varies from the PHDapproach that maps the reference simulation up to the maximal value. A possible explanation for that trend is the extraction method of the load-pull approaches. For the QPHDapproach, several values of the amplitude $\hat{u}_{22}$ should be used in addition to the phase sweep in order to get a better conditioned matrix $\mathbf{A}$ which will be performed in future work.

\section{Conclusions}

In this contribution, different approaches of the $X$ parameters were examined in order to model load-dependent behavior of nonlinear transfer systems considered as multiports. In addition to the $X$-parameter extracted at each load by means of fundamental frequency load-pull technique (Root et al., 2013), the model order reduced approaches of Cai et al. (2015) and Cai and Yu (2015) were compared. On the basis of an exemplary simulation, it was shown that the model order reduced approaches can be a good compromise if used the vicinity of the system situation for which they were extracted. For large deviation from that state, they can introduce a large error due to the extraction methods based on the numeric least-squares approximation. In addition, the randomized phase variation of the load-pull tuner is not ca-

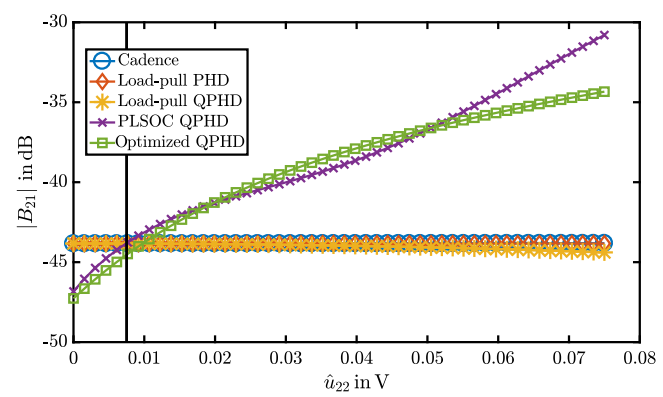

Figure 5. Behavior of $\left|B_{21}\right|$ as a function of $\hat{u}_{22}$ at $\left|\Gamma_{21}\right|=0.65$ and $\left|\Theta_{21}\right|=135^{\circ}$.

pable to extract $X$-parameter for small signals at the input port $A_{1 k}$.

Data availability. No data sets were used in this article.

Competing interests. The authors declare that they have no conflict of interest.

The publication of this article was funded by the open-access fund of Leibniz Universität Hannover.

Edited by: J. Anders

Reviewed by: two anonymous referees

\section{References}

Cai, J. and Yu, C.: A new extraction method of nonlinear behavioral model for RF power transistor, in: vol. 2, 2015 Asia-Pacific Microwave Conference (APMC), 6-9 December 2015, Nanjing, China, 1-3, https://doi.org/10.1109/APMC.2015.7413226, 2015.

Cai, J., King, J. B., Zhu, A., Pedro, J. C., and Brazil, T. J.: Nonlinear Behavioral Modeling Dependent on Load Reflection Coefficient Magnitude, IEEE T. Microw. Theory Tech., 63, 1518-1529, https://doi.org/10.1109/TMTT.2015.2416232, 2015.

Dahmen, W.: Numerik für Ingenieure und Naturwissenschaftler Springer, Berlin, Heidelberg, 2008.

Ghannouchi, F. M. and Hashmi, M. S.: Load-Pull Techniques with Applications to Power Amplifier Design, in: Springer Series in Advanced Microelectronics, Springer, Dordrecht, New York, 2013.

Gonzalez, G.: Microwave Transistor Amplifiers: Analysis and Design, 2nd Edn., Prentice Hall, Upper Saddle River, NJ, 1997.

Howell, K.: Principles of Fourier analysis, Chapman \& Hall/CRC, Boca Raton, Florida, 2001.

Root, D. E., Verspecht, J., Horn, J., and Marcu, M.: X-Parameters: Characterization, Modeling, and Design of Nonlinear RF and Microwave Components, in: The Cambridge RF and Microwave Engineering Series, Cambridge University Press, Cambridge, 2013.

Verspecht, J. and Root, D. E.: Polyharmonic distortion modeling, IEEE Microw. Mag., 7, 44-57, https://doi.org/10.1109/MMW.2006.1638289, 2006. 